\title{
Identifying the Research Needs of Four Large High- Technology Companies
}

\author{
Mehmet Akşit ${ }^{1}$, Bedir Tekinerdoğan ${ }^{2}$, Hasan Sözer ${ }^{3}$, Hakan Faruk Safi ${ }^{4}$, Meryem Ayas ${ }^{4}$
}

\begin{abstract}
Software excellence is defined as the adoption of advanced software techniques and methods to cope with the complexity and the needs of modern software systems. To accomplish this objective, the companies that develop software intensive systems must be able to follow and adopt the state-ofthe-art developments in computer science and technology. In general, this implies carrying out research activities with the relevant universities. This paper presents an overview of a pilot implementation of the approach termed as "Describe, Search and Acquire the Required Capability" (DESARC), which aims at establishing a more effective identification of research projects, allocation of these research projects to research groups and the cooperation between universities and industry. In our previous publication [1], we have presented the initial results of the method. Currently, we have extended this study and applied it in total to four large high-technology companies. This paper can be useful for the strategic developers who are interested in setting up university-industry cooperation and for the researchers who want to have an industrial view of the trends on computer science and software engineering.
\end{abstract}

Keywords - university-industry cooperation, the DESARC method, trends in computer science and software engineering.

\section{Introduction}

Companies which need to adopt cutting edge technologies to produce high-technology products must follow the actual developments in science and technology.

Since software is the key enabler of many high-technology products, advancements in computer science and software engineering play an important role in producing higher quality software intensive products. Unfortunately, companies have very often difficulties to follow the advancements effectively due to tight production plans and limited budget for research. In [1], we have introduced a new way for efficient universitysoftware industry cooperation called DESARC and summarized our findings..

\footnotetext{
${ }^{1}$ Department of Computer Science, University of Twente

Enschede, The Netherlands

${ }^{2}$ Chair of Information Technology, Wageningen University

Wageningen, The Netherlands

${ }^{3}$ School of Engineering, Özyeğin University

Istanbul, Turkey

${ }^{4}$ STM Savunma Teknolojileri Mühendislik ve Ticaret A.Ş. Ankara, Turkey
}

Conclusions in [1] were limited to a visit of two companies only. Since then we have visited in total four large hightechnology companies, and based on the strategic objectives of the companies we defined in total $150 \mathrm{Ph}$.D. projects in detail.

This paper describes the outcome and extensions to the DESARC method, gives information about the product domains of the companies that were analysed, summarizes the characteristics of the research projects defined, makes statements about the observed trends in computer science and software engineering, and finally describes a framework to execute the research projects in an efficient and effective manner.

This paper is organized as follows. The following section explains the DESARC method briefly. The third section gives information about the kinds of products the four companies are manufacturing. A classification of the research projects are given in section four. Based on the defined research projects, section five makes statements about the observed trends in computer science and software engineering. Section six defines a cooperation framework that will be used in managing the research projects. Finally, section seven gives lessons learned and conclusions.

\section{Overview of the DESARC Method}

In this section, we will give a short overview of the adopted methodology. For a more detailed information, the readers must refer to [1]. Fig. 1 shows the sub-processes of the DESARC method. Contrary to traditional approaches, company stakeholders play here an important role in the identification of the research topics. Nevertheless, although the company manages the process, a team of academic consultants jointly carries out the assessment and research definition activities. In addition, the grant giving agencies strongly cooperate with the overall process.

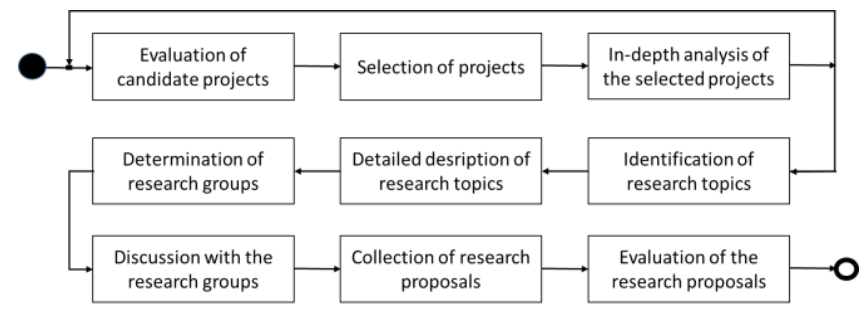

Fig. 1 The sub-processes of the DESARC method. For simplicity, not all possible iterations are shown. 
As the first step, a set of candidate software development projects are evaluated. In this stage, the strategically important projects are considered.

As the second step, a set of development projects are selected for further consideration.

As the third step, meetings are organized with the software development teams of the company to identify and describe the required expertise and consequently the necessary research projects.

In the fourth step, a set of research topics are identified. report.

In the fifth step, the research topics are described in a

In the sixth step, the relevant university researchers, called principle investigators, who have the required expertise for the intended research activities are determined and contacted.

As the remaining steps, after various discussions with the researchers, collection of the final research proposals (called technical annex) is written by the selected principle investigators, the projects are evaluated and prioritized, and reported to project funding organizations which finance the projects if the budget is feasible.

\section{The Product Domain Categories}

To give an idea about the products that are produced, in the following we list the kinds of product domain categories for each company. Each company stated its interest in manufacturing products in these product domains in the coming decade. Furthermore, for each product domain, we give the number of identified and defined Ph.D. projects.

\section{Company 1:}

- On-board systems (14 Ph.D.'s);

- Simulation systems (10 Ph.D.’s);

- Mission critical enterprise systems (14 Ph.D.'s);

- Mission critical systems of systems (10 Ph.D.'s);

Company 2:

- Avionics systems (15 Ph.D.'s);

- Communication systems of systems (9 Ph.D.'s);

- Mission-critical hybrid control systems (6 Ph.D.'s);

- Sensor fusion systems (3 Ph.D.'s);

Company 3:

- Intelligent platforms (8 Ph.D.'s);

- Distributed simulation (6 Ph.D.'s);

- Intelligent control systems (6 Ph.D.'s);

\section{Company 4:}

- Modernization of flight systems (11 Ph.D.'s);

- Satellite systems (14 Ph.D.'s);

- Unmanned aerial systems (17 Ph.D.'s);

- Generic fault tolerant computing (2 Ph.D.'s);

- Generic fault-removal techniques (5 Ph.D.'s);

\section{The Category of Research Projects}

All the Ph.D. projects (150 in total) have been categorized with respect to their research content. In the following, we present these categories and indicate the approximate percentage of the research effort per category.

Adaptability and reuse (25\%): In general adaptability and reuse refer to product-line engineering, object-oriented, component-oriented, aspect-oriented and event-based languages, domain specific languages, compositional techniques, and model-driven engineering techniques.

Model-based verification (24\%): By means of model-based verification, one or more of the following techniques are meant: model-checking, model-based testing, static and dynamic analysis, and runtime verification.

Domain-specific architectures (12\%): Domain-specific architectures are fault-tolerant architectures, secure architectures, event-driven architectures, self-adaptive and self-healing architectures, real-time systems, sensor systems, cyber-physical systems and systems of systems.

Domain-specific languages (10\%): All kinds of domainspecific languages are required to be researched for addressing the mission-critical and safety-critical tasks.

Design methods (8\%): In this context design methods aim to integrate the adopted techniques in an efficient and effective manner.

System design (6\%): These research activities refer to all kinds of studies which relate to designing distributed infrastructures.

Model building (6\%): These research activities refer to application-specific continuous and/or discrete models.

Data/cloud management (3\%): High speed mission and safety critical cloud and data management research activities are considered in this context.

Application frameworks (3\%): These research activities refer to building application specific libraries and design environments.

Architecture assessment (3\%): Analytical and empirical assessments of new architectures are considered by these projects. 
The percentage of a required work does not necessarily refer to the relative importance of a work.

\section{v. The Trends in Computer Science and Software Engineering}

The identified and defined projects were driven from the strategic needs of the companies and there was a consensus over the content of the projects among the technical persons working at these companies. By inspecting the projects, we could identify the following trends:

- Product-line instead of product design; (system product-lines, multiple-product-lines, etc.);

- Systems of systems instead of systems perspective;

- Ecosystem design instead of distributed system design;

- Ecosystem design instead of service-oriented system design;

- Event-based semantic interaction instead of message passing;

- Modularization of semantic behaviour instead of data abstractions and functional abstractions;

- Auto-adaptive control architectures instead of traditional modules (components) for the basic building blocks;

- Distributed problem solving instead of centralized problem solving;

- Domain specific languages (DSL's) instead of general purpose programming languages;

- Embedded domain specific languages (EDSL's) instead of collection of DSL's;

- Declarative EDSL's instead of imperative EDSL's;

- Uniform integration of an open-ended set of both compositional techniques (object, aspect and event compositions) and transformational (modeldriven engineering) techniques instead of a fixed set of language and system specific techniques;

- Multi-level multi-dimensional closure property in compositions instead of single-level, hierarchical composition;

- Embedded multi-objective optimizations instead of ad-hoc hand-crafted single objective optimization;

- Uniform integration of model-based verification techniques (model-checking, static and dynamic analysis, run-time verification, model-based testing and fault-tolerance techniques) and programming environments instead of independent tool-specific verification approaches.
- Active (generative) pattern-based libraries that integrate the above listed properties with integrated heuristic management instead of traditional language-specific passive libraries.

\section{vi. Executing the Research Projects in a University-Industry Cooperation Framework}

In order to maximise the possible benefits of the research projects it is found necessary to consider the following characteristics of the defined Ph.D. projects:

Relevancy: Since all research projects have been defined in close cooperation with the company managers and engineers, they are considered highly relevant.

Alignment with the current state-of-the-art research: Since the research projects are to be carried out with a selected set of universities, the content of the projects were discussed with the principal investigators of the candidate universities.

Cross-fertilization: Many research activities can benefit from each other. To maximize the benefit, it is found necessary to strongly coordinate the related projects with each other.

Industry-as-laboratory: To identify the relevant problems and to test the proposed solutions, it is found necessary that the principle investigators and the affiliated (Ph.D. and/or M.Sc., etc.) students visit the companies regularly and carry out experiments within industrial context. To this aim, companies must provide personal assistance and industrial case studies.

Academic research steering committee: To coordinate the activities effectively and efficiently, it is decided to set up a central organization. There will be nevertheless a separate steering committee for each company and for each strategic topic in that company. Of course, human and other resources can be shared among these committees if appropriate.

Integration with a technology maturity improvement process: The ultimate purpose for industrial research activities is to enhance the technological level of the companies, to turn technology into economic values and to make the company's successful businesses in a sustainable way. For this purpose, a model is defined called Software Technology Maturity Model [2]. The activities of the academic research steering committees will be integrated within the process of a technology maturity improvement process. The process will be financed by governmental grant-giving organizations.

\section{vII. Conclusion}

In this paper we have presented an approach for enhancing the research capability of high-technology companies. The approach is based on the "Describe, Search and Acquire the 
Required Capability" (DESARC) method. The practical realization of the research projects is based on the so-called demand-driven industry-as-laboratory approach in which academic institutes collaborate with the industry to identify relevant research problems and derive the required solutions. The DESARC method includes the identification of the research problems, the description of research projects, and the selection of research groups who are experts in the selected domains of the research projects. We have applied the method to four large high-technology companies from January 2014 to February 2016.

Based on the analysis of the existing projects of four companies and several meetings we were able to identify 150 $\mathrm{PhD}$ projects. Each research project has been described in detail. The companies have agreed on the content of these reports. These reports also indicate a clear trend in computer science and engineering for the coming decade. This trend is listed in section five.

Based on these successful results, we are currently setting up a cooperation framework, sponsored by the Turkish Government.

We are confident that since general-purpose proposal selection processes are eliminated and the research definition activities are targeted to the company needs, the DESARC method causes much less overhead. In addition, the method results in the identification of the relevant technological problems and as such the related research activities will likely to have a higher degree of technology transfer than the traditional grantproposal based research approaches $[3,4]$.

\section{References}

[1] M. Akşit, B. Tekinerdogan, H. Sözer, H.F. Safi, and M. Ayas. "The DESARC method: An effective approach for university-industry cooperation". In: Proceedings of the International Conference on Advances in Computing, Control and Networking, ACCN 2015, 3-4 Jan 2015, Bangkok, Thailand. pp. 51-53. Institute of Research Engineers and Doctors. 2015.

[2] M. Akşit, H. Sözer, B. Tekinerdogan, H.F. Safi, and M. Ayas, "SETMM Software engineering technology maturity model", University of Twente technical report. 2016.

[3] Y. Caloghirou, A. Tsakanikas and N. S. Vonortas, "University-industry cooperation in the context of the European framework programmes", Journal of Technology Transfer, 26, 153-161, 2001

[4] R. Teigland and A. Schenkel, "The impact of EU framework projects on competitiveness", [The Tenth Annual SNEE European Integration Conference, Mölle 2008]. 